\title{
EVOKED POTENTIALS IN TYPE I DIABETIC CHILDREN AND THEIR RELATION WITH SYNCHRONOUS BLOOD GLUCOSE, SERUM AND INTRACELLULAR MAGNESIUM AND GLYCOSYLATED HAEMOGLOBIN LEVELS
}

\author{
Y. Karal, S. Karasalihoğlu , N. Turgut , N. Oner , F. Tütüncüler \\ Trakya University Faculty of Medicine, Paediatric Neurology, Edirne, Turkey \\ Namık Kemal University Faculty of Medicine, Neurology, Tekirdağ, Turkey \\ Memorial Hospital, Pediatrics, İstanbul, Turkey \\ Trakya University Faculty of Medicine, Paediatric Endocrinology, Edirne, Turkey
}

Background and Aims:Type I Diabetes Mellitus is a chronic disease associated with high morbidity and complications. The aim of this study was to evaluate central nervous system involvement by evoked potentials in diabetic children and identify the relation with metabolic disturbances.

Methods: The study comprised of 31 children with Type I diabetes (at least 1 year follow up with Tip 1 Diabetes and had no other chronic diseases) aged 5-14 years (10 male, 21 female ) and 35 age and sex matched non-diabetic and healty children (14 male, 21 female). The patients who had retinopathy and nefropathy and other vasculer disorders were excluded from the study. The diabetic group were getting $1 \mathrm{IU} / \mathrm{kg}$ insülin daily. Pattern visual evoked potentials (VEP), brainstem auditory evoked potentials (BAEP), somatosensory evoked potentials (SEP) and transient evoked otoacoustic emissions were recorded in all subjects, and metabolic control was evaluated after 8 hour fasting in terms of glyceamia, glycosylated haemoglobin, serum and intracellular magnesium status.

Patients were divided into subgroups according to glycosylated haemoglobin as $\mathrm{H} 1-\mathrm{H} 2(\mathrm{HbA} 1 \mathrm{c}<9 \%-\mathrm{HbA} 1 \mathrm{c} \geq 9 \%)$, synchronous blood glucose as KŞ1-KŞ2 (blood glucose <250 $\mathrm{mg} / \mathrm{dl}$-blood glucose $\geq 250 \mathrm{mg} / \mathrm{dl}$ ), serum magnesium levels as M1-M2 (Mg<1.8mg/ml-Mg $\geq 1.8 \mathrm{mg} / \mathrm{ml})$, intracellular magnesium levels as HM1-HM2 ( $\mathrm{Mg} \leq 4 \mathrm{ng} / \mathrm{dl}-\mathrm{Mg}>4 \mathrm{ng} / \mathrm{dl}$ ) and duration of diabetes as $\mathrm{S} 1$-S2 ( $<48$ months- $\geq 48$ months).

Results: In the diabetic group, serum magnesium levels were low whereas there was no difference in intracellular magnesium levels when compared with non-diabetic subjects (Table 1). There were significant abnormalities in the latency values of visual evoked potentials in the diabetic group as a whole (Table 2), $\mathrm{H} 1-\mathrm{H} 2$ subgroups, and also its $\mathrm{HM} 1$ and $\mathrm{S} 2$ subgroups. There was no statistical significance between groups in somatosensory evoked potential latencies except M1 subgroup. The latencies of brainstem auditory evoked potential waves were signicantly impaired in diabetic subjects, especially in $\mathrm{H} 2, \mathrm{M} 1$ and $\mathrm{S} 2$ subgroups. Peripheral transmission time (wave I) was signicantly delayed while I-V and III-V intervals were shorter (Table 3). There was no significant difference between groups in emission values.
Table 1. Biochemical parameters of groups.

\begin{tabular}{|c|c|c|c|}
\hline & Diabetic group & Non-diabetic group & $\mathrm{p}$ \\
\hline HbA1c (\%) & $9.37 \pm 2.11(5.90-13.60)$ & $4.79 \pm 0.76(3.60-7.60)$ & $<0.001^{*}$ \\
\hline $\begin{array}{c}\text { blood glucose } \\
(\mathrm{mg} / \mathrm{dl})\end{array}$ & $209 \pm 98(48-380)$ & $89 \pm 8(71-107)$ & $<0.001^{\dagger}$ \\
\hline $\begin{array}{c}\text { serum } \\
\text { magnesium } \\
\text { (mg/ml) }\end{array}$ & $1.77 \pm 0.27(1.12-2.34)$ & $2.01 \pm 0.20(1.60-2.50)$ & $<0.001^{\dagger}$ \\
\hline $\begin{array}{c}\text { intracelluler } \\
\text { magnesium } \\
\text { (ng/dl) }\end{array}$ & $4.13 \pm 0.91(1.10-5.53)$ & $4.23 \pm 0.50(2.86-5.50)$ & $\mathrm{ns}$ \\
\hline
\end{tabular}

Results; mean \pm SD (min-max) . ns: Non significant *Student T Test, ${ }^{\dagger}$ Mann-Whitney U Test

Table 2. Comparison of VEP Latencies and Amplitudes between groups.

\begin{tabular}{|c|c|c|c|}
\hline Waves & Diabetic group $(n=31)$ & $\begin{array}{c}\text { Non-diabetic group } \\
(n=35)\end{array}$ & $p$ \\
\hline $\begin{array}{c}\text { P 100 latencies } \\
(\mathrm{ms})\end{array}$ & $108.50 \pm 4.78(99.90-125.10)$ & $106.80 \pm 3.88(97.20-114.30)$ & $<0.05^{*}$ \\
\hline $\begin{array}{c}\text { P } 100 \\
\text { amplitudes } \\
(\mu \mathrm{V})\end{array}$ & $19.25 \pm 7.21(6.00-37.60)$ & $24.40 \pm 9.05(9.40-43.50)$ & $<0.001^{\dagger}$ \\
\hline
\end{tabular}

Results; mean \pm SD (min-max) . ns: Non significant * Student T Test, ${ }^{+}$Mann-Whitney U Test

Table 3. Comparison of BAEP Latencies between groups

\begin{tabular}{|c|c|c|c|}
\hline \multirow[b]{2}{*}{ WAVES } & \multicolumn{2}{|c|}{$\operatorname{WAVES}(\mathrm{ms})$} & \multirow[b]{2}{*}{$\mathrm{p}$} \\
\hline & Diabetic group $(n=31)$ & $\begin{array}{l}\text { Non-diabetic group } \\
(\mathrm{n}=35)\end{array}$ & \\
\hline Wave I & $1.70 \pm 0.15(1.40-2.10)$ & $1.65 \pm 0.08(1.50-1.90)$ & $<0.05^{\star}$ \\
\hline Wave III & $3.74 \pm 0.19(3.36-4.16)$ & $3.77 \pm 0.12(3.54-4.08)$ & ns \\
\hline Wave V & $5.60 \pm 0.28(4.06-6.14)$ & $5.65 \pm 0.15(5.32-5.98)$ & ns \\
\hline Wave interval I-III & $2.03 \pm 0.15(1.68-2.40)$ & $2.10 \pm 0.13(1.72-2.38)$ & $<0.05^{*}$ \\
\hline Wave interval III-V & $1.89 \pm 0.13(1.62-2.36)$ & $1.88 \pm 0.14(1.52-2.26)$ & ns \\
\hline Wave interval I-V & $3.92 \pm 0.17(3.54-4.52)$ & $3.99 \pm 0.17(3.62-4.40)$ & $<0.05^{\dagger}$ \\
\hline
\end{tabular}

Results; mean \pm SD (min-max) . ns: Non significant ${ }^{*}$ Student T Test, ${ }^{\dagger}$ Mann-Whitney U Test

Conclusion: Central nervous system involvement, highly related with glycosylated haemoglobin, serum and intracellular magnesium levels but not with synchronous blood glucose, may be the earliest complication in diabetes mellitus that can be detected by evoked potentials. We consider that these measurements will be useful in the routine follow up of diabetes mellitus. 